\title{
High prevalence of systemic disease and mortality in Asian subjects with systemic lupus erythematosus
}

\author{
A Samanta, J Feehally, S Roy, F E Nichol, P J Sheldon, J Walls
}

\begin{abstract}
All patients with systemic lupus erythematosus (SLE) (American Rheumatism Association criteria with positive antinuclear antibody titre) and who attended any of the three general hospitals in Leicester over a 10 year period were ascertained using several complementary sources. Eighty seven subjects (26 Asian, 61 white) were identified. The estimated prevalence of SLE in Leicester is $0.4 / 1000$ for Asian and $0.2 / 1000$ for white subjects. Mean age of onset of the disease was 24 years in Asian and 31 years in white subjects, with both groups showing a female preponderance. Proteinuria $(>1 \mathrm{~g} / 24 \mathrm{~h})$ was noted in $15(58 \%)$ Asian and 21 (35\%) white subjects; neuropsychiatric disease in $10(38 \%)$ Asian and $8(13 \%)$ white subjects; myalgic symptoms with raised muscle enzymes in 9 (35\%) Asian and 3 (5\%) white subjects. Nineteen (73\%) Asian subjects were positive for extractable nuclear antigens as well, at some stage of their disease, compared with $6(10 \%)$ white subjects. Immunosuppressive treatment was required in $12(46 \%)$ Asian and $12(20 \%)$ white subjects, and deaths of seven Asian and five white subjects were attributed to SLE.

These findings show that Asian subjects have a higher prevalence of SLE with greater systemic disease and mortality.
\end{abstract}

Systemic lupus erythematosus (SLE) is known to have diverse clinical manifestations and in part this may be related to ethnic origin. ${ }^{1}$ The Chinese population in Singapore have a greater degree of skin disease and tend to show greater neurological abnormalities with time. ${ }^{2}$ The Melanesian population in New Zealand have a mortality from SLE which is six times higher than that of the indigenous white population. Renal and neurological disease also tend to occur more commonly in this group. ${ }^{3}$ The Tokyo study showed that Japanese subjects have a lower incidence of skin and joint disease and leucopenia. ${ }^{4}$ These studies serve not only to emphasise the varying features of SLE but also to suggest that ethnic factors may be of importance in the expression and the severity of the disease. We studied the clinical features of SLE in migrants to the United Kingdom from the Indian subcontinent (Asian subjects) and compared them with those of the indigenous population (white subjects) over a 10 year period.

Patients and methods

Patients satisfying the diagnostic criteria for
SLE who were resident in Leicester and had presented between 1979 and 1988 were identified.

To ensure that ascertainment was as complete as possible information was compiled from several complementary sources: (1) Hospital activity analysis was scrutinised for the three general hospitals of Leicester, which serve the total population of Leicestershire. All diagnoses of SLE, connective tissue disease, or vasculitis were considered. (2) Histological reports from a centralised pathology laboratory were examined, particularly reports on kidney and skin biopsies, for a diagnosis of SLE, connective tissue disease, or vasculitis. (3) Death certification records were examined for the inclusion of SLE or connective tissue disease as a cause of death. (4) Individual doctors in Leicester were asked to provide a list of patients with SLE or connective tissue disease under their care.

Subsequently, case notes of all patients were examined to confirm an abnormally raised titre of antinuclear antibody plus at least three other criteria-that is, at least four of the American Rheumatism Association revised criteria 1982. Case notes were scrutinised to collect demoraphic details of subjects and to record the clinical features of SLE which might have occurred at any time since presentation. Clinical and laboratory features recorded were those of malar rash, discoid rash, photosensitivity, oral ulcers, arthralgia, pleurisy, pericarditis, haematuria, urinary casts, proteinuria ( $24 \mathrm{~h}$ urine collection measured at $>1 \mathrm{~g}$ ), recorded neurological deficit on clinical examination, reported psychiatric abnormality, myalgic symptoms with recorded muscle weakness, maximum activity of creatine kinase, highest antinuclear antibody titre, presence of extractable nuclear antigens, hypocomplementaemia, DNA binding, and positive titre double stranded DNA. Asian patients were identified by name.

Statistical analysis was performed by $\chi^{2}$ test with Yates's correction and statistical significance only at, or greater than, the $5 \%$ level is shown.

\section{Results}

Eighty seven patients with SLE were ascertained (26 Asian, 61 white). There were more women in both groups, though the proportion of men was greater in the Asian group $(p<0.01)$. The mean age of onset of symptoms was lower and the mean duration of symptoms longer in the Asian group (table).

There were no statistical differences in mucocutaneous features, arthralgia, or pleuro- 
Details of subjects studied. Results are given as means (SE)

\begin{tabular}{|c|c|c|}
\hline & $\begin{array}{l}\text { Asian } \\
(n=26)\end{array}$ & $\begin{array}{l}\text { White } \\
(n=61)\end{array}$ \\
\hline $\begin{array}{l}\text { Female:male } \\
\text { Age onset (years) }\end{array}$ & $\begin{array}{l}18: 8 \\
24(6)\end{array}$ & $\begin{array}{l}53: 8 \\
31(5)\end{array}$ \\
\hline $\begin{array}{l}\text { Duration of symptoms before } \\
\text { presentation (months) } \\
\text { Duration of follow up (years) }\end{array}$ & $\begin{array}{l}15(10) \\
8(0 \cdot 5)\end{array}$ & $\begin{array}{l}6(6) \\
8 \cdot 2(0 \cdot 5)\end{array}$ \\
\hline
\end{tabular}

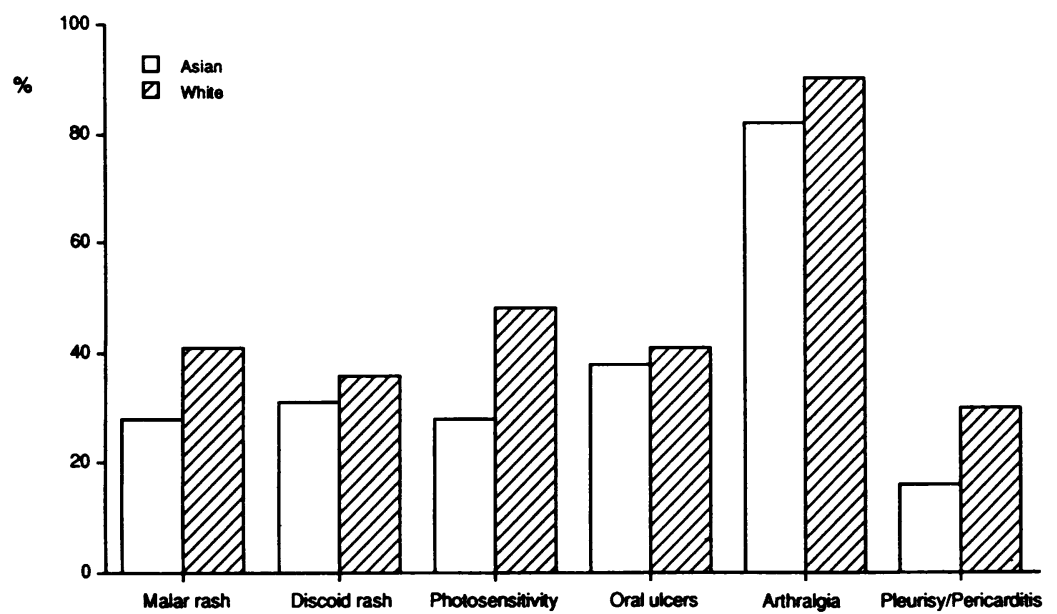

Figure 1 Mucocutaneous, pleuropericarditic, and joint manifestations of sytemic lupus erythematosus in Asian and white subjects.

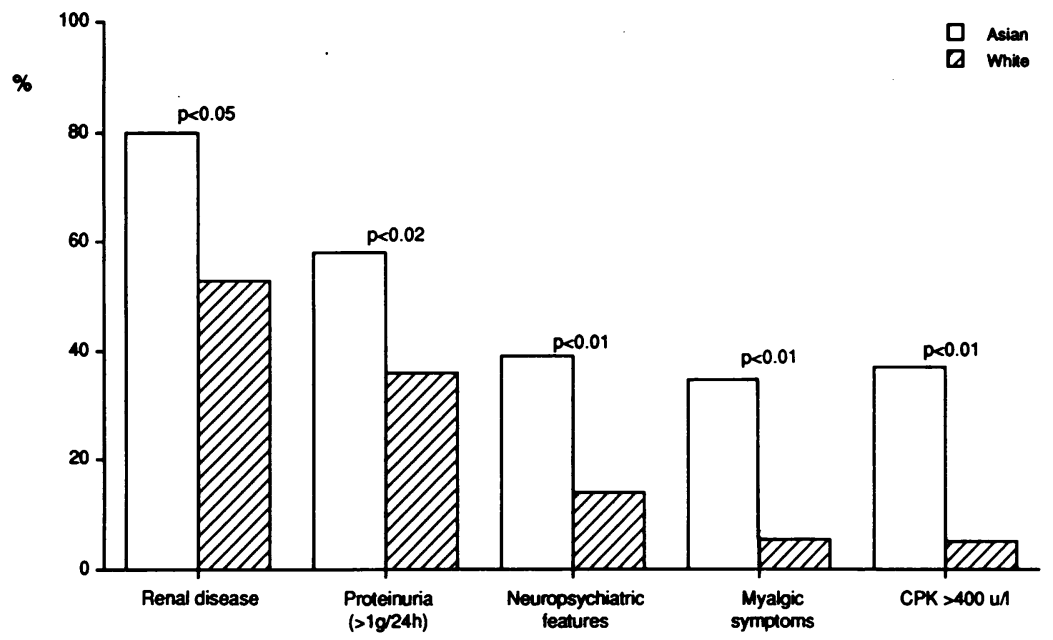

Figure 2 Renal and neuropsychiatric manifestations of systemic lupus erythematosus in Asian and white patients. $C P K=$ creatine kinase.

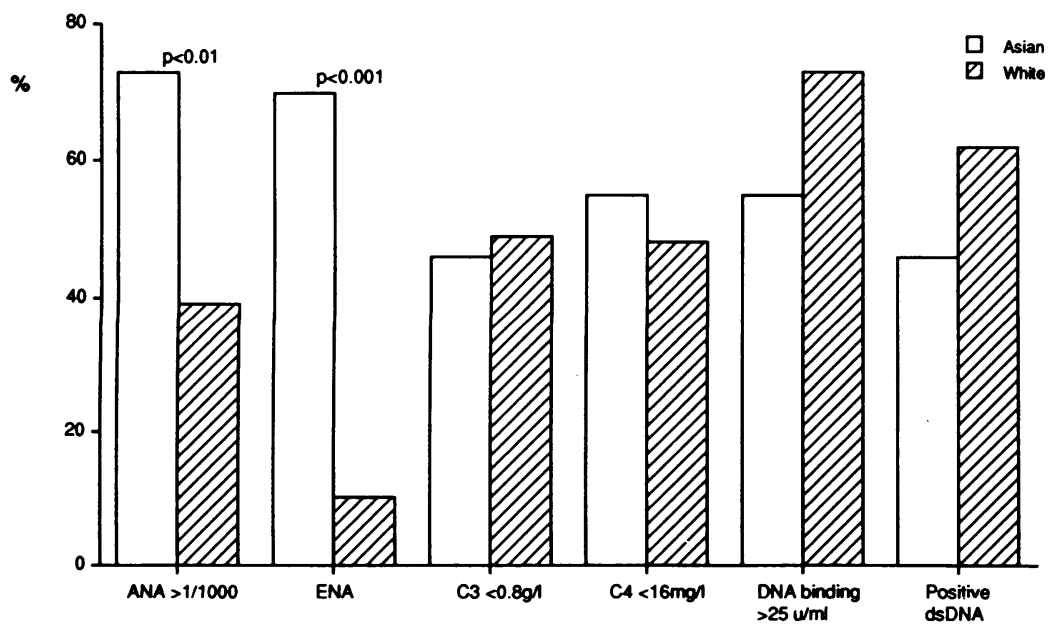

Figure 3 Serological features of systemic lupus erythematosus in Asian and white patients. $A N A=$ antinuclear antibody; $E N A=$ extractable nuclear antigens. pericarditis between Asian and white subjects (fig 1). Asian patients had significantly higher renal disease (haematuria or casts) and proteinuria (fig 2); and a higher prevalence of extractable nuclear antigens and a raised titre of antinuclear antibodies, greater than 1/1000 (fig 3).

Twenty four (92\%) Asian patients compared with $46(75 \%)$ white subjects were treated with steroids at some stage of the disease. A higher proportion of Asian patients needed immunosuppressive treatment $(12(46 \%) v 12(20 \%)$; $\mathrm{p}<0.05)$. There were seven deaths due to active SLE or related causes in the Asian group compared with five in the group of white subjects (Fisher's exact test $p=0.04$ ).

\section{Discussion}

Several studies have suggested that the clinical features of SLE may vary in different ethnic groups. ${ }^{1-4}$ Studies from the United States of America have shown that SLE in black Americans is more aggressive than that seen in white Americans. ${ }^{6}$ There is some suggestion, however, that this may be related to social class and whether or not medical care is funded by public or private sources. ${ }^{7}$ In our study socioeconomic status could not be ascertained. It is also known, however, that SLE in the oriental (Far Eastern) population in North America is more aggressive and has a greater mortality and that these differences persist even after adjusting for variables which may affect them. ${ }^{8}$ These studies suggest that although environmental factors may to some extent be responsible for the severity of the disease, there are inherent features within different populations which by themselves may account for some of the differences.

Our study is retrospective, and therefore has some of the shortcomings that are common to all studies of this nature. Patients were seen at different times over a 10 year period and clinical features were recorded by different doctors over the study period. There may arguably be a certain degree of heterogeneity in the process of documentation. This would apply equally to both the groups studied, however, and would therefore minimise any bias.

The question also arises as to whether there may be any differences in the referral patterns from the general practices that Asian subjects normally attend. From past epidemiological work done in Leicester and from informal discussions with general practitioners it would seem that there are no major differences in referral patterns, and that most would tend to refer sooner rather than later. ${ }^{10}$ It is noteworthy that the duration of symptoms before presentation was slightly longer in the Asian group and the mean duration of follow up slightly less, though these differences did not reach statistical significance. A population based estimate suggests that the prevalence of SLE in Leicester is about $0.4 / 1000$ in Asians compared with $0 \cdot 2 / 1000$ in whites. The higher prevalence in Asians is of interest and needs to be explored further.

All patients in this study strictly fulfilled the 
American Rheumatism Association diagnostic criteria for SLE, thus forming a diagnostically homogeneous group. All clinical and laboratory information was collected in a standardised fashion for both groups. The higher incidence of renal disease would not reflect a bias due to inclusion of patients known to the nephrology services in Leicester because patients in this study were under the care of a range of clinical disciplines. The higher incidence of reported proteinuria in excess of $1 \mathrm{~g} / 24 \mathrm{~h}$, in addition to other urinary abnormalities, provides quantita-tive evidence of a higher incidence of renal disease in Asian subjects with SLE.

Neuropsychiatric and myalgic features also occurred more commonly in Asians and were supported by clinically recorded abnormal neurology and a high activity of creatine kinase. Specificities of extractable nuclear antigens and an assessment of Sjögren's syndrome (which may at times mimic SLE) could not be ascertained, but Asian subjects tended to show a clustering of myalgia, arthralgia, neuropsychiatric features, and a raised antinuclear antibody titre early in the course of their disease.

Studies on SLE from India ${ }^{11}$ seem to suggest that the overall pattern of the disease is similar to that seen in the West. Difficulties in interpreting such data may occur owing to differences in the method of data collection. This study has overcome this by collecting data in a standardised fashion in two different ethnic groups geographically located in the same area. Asian subjects with SLE have a higher prevalence of systemic illness with renal and neuromuscular disease. The disease is clinically more aggressive with a greater need for immunosuppressive treatment, and a greater mortality. The cause for this needs to be elucidated.

We are grateful to the late Dr Andrew Campbell, consultan immunopathologist for his helpful discussions; to medica records staff for data retrieval; and to Ms Judith Noble for her secretarial assistance.

1 Fries J F, Holman H R. Systemic lupus erythematosus: a clinical analysis. In: Smith L H Jr, ed. Major problems in internal medicine. London: Saunders, 1975: 8-47.

2 Feng $\mathbf{P} \mathbf{H}$, Boey M L. Systemic lupus erythematosus in Chinese. The Singapore experience. Rheumatol Int 1982; 2 : $151-4$.

3 Hart H H, Grigor R R, Caughey D E. Ethnic differences in the prevalence of systemic lupus erythematosus. Ann the prevalence of systemic

4 Yokohari R, Tsunematsu T. Application, to Japanese patients, of the 1982 American Rheumatism Association revised criteria for the classification of systemic lupus erythematosus. Arthritis Rheum 1985; 28: 693-8.

5 Tan E M, Cohen A S, Fries J F, et al. The 1982 revised criteria for the classification of systemic lupus erythematosus. Arthritis Rheum 1982; 25: 1271-7.

6 Ginzler E M, Diamond H S, Weiner M, et al. A multicentre study of outcome in systemic lupus erythematosus. I. Entry variables as predictors of prognosis. Arthritis Rheum 1982, 25: 601-11.

7 Rosner S, Ginzler E M, Diamond H S. A multicentre study of outcome in systemic lupus dro. A multicentre study of outcome in systemic lupus erythemato
death. Arthritis Rheum 1982; 25: 612-7.

8 Kaslow R A. High rate of death caused by systemic lupus erythematosus among U.S. residents of Asian descent. Arthritis Rheum 1982; 25: 414-9.

9 Samanta A, Burden A C, Feehally J, Walls J. Diabetic renal disease: differences between Asians and white Caucasians. BMF 1986; 293: 366-7.

10 Samanta A, Roy S. Referrals from general practice to rheumatology clinic. $\mathrm{Br} \mathcal{F}$ Rheumatol 1988; 27: 74-6.

11 Malaviya A N, Misra R, Baneriee S, et al. Systemic lupus erythematosus in North India Asians. A prospective erythematosus in North India Asians. A prospective Int 1986; 6: 97-101. 